\title{
Oxigenoterapia domiciliar em crianças: relato de sete anos de experiência"
}

\author{
Helena Teresinha Mocelin ${ }^{1}$, Gilberto Bueno FisCHER ${ }^{2}$, Lilian CRistine RAnZi ${ }^{3}$, \\ RosÂngela Dias RosA ${ }^{4}$, MARIA REGINA PHILOMENA ${ }^{5}$
}

Objetivo: Descrever os resultados de uma experiência de sete anos com uso de oxigenoterapia domiciliar em crianças com hipoxemia decorrente de doença pulmonar crônica. Pacientes $\boldsymbol{e}$ métodos: É um estudo retrospectivo e descritivo realizado no Hospital de Crianças Santo Antonio, de Porto Alegre, Rio Grande do Sul, na região Sul do Brasil. No geral, avaliaram-se as características das crianças com oxigenoterapia domiciliar acompanhadas durante o período de janeiro de 1993 a janeiro de 2000. Resultados: Dos 40 pacientes estudados, 29 (72,5\%) eram meninos. A idade de início do

oxigênio no domicílio variou de dois meses a 13 anos e seis meses, sendo que 18 (45\%) eram

menores de um ano (média: 24,8 meses; mediana: 13 meses). As enfermidades mais prevalentes foram: bronquiolite obliterante, 31 (77,5\%); pneumopatia obstrutiva crônica secundária à aspiração, três; hipogamaglobulinemia, dois; pneumopatia supurativa crônica, dois; proteinose alveolar e tuberculose, um caso cada. O tempo da última internação antes de iniciar oxigênio domiciliar variou de nove a 240 dias (média: 64 dias; mediana 50 dias). A maioria dos pacientes recebeu oxigênio por aparelho concentrador, 30 (75\%), e os demais, por cilindro. O tempo de uso variou de 27 a 1.620 dias, mediana de 392,5 dias. Ocorreram cinco óbitos por complicações da doença de base e um por retirada acidental da traqueostomia. Dos 19 pacientes em que o oxigênio foi suspenso, o diagnóstico era bronquiolite obliterante em 15, pneumonia aspirativa em dois e tuberculose e proteinose alveolar um caso cada. Conclusão: Apesar da necessidade de oxigênio por períodos prolongados, a evolução foi satisfatória na maioria das crianças. Oxigênio domiciliar para crianças oxigenodependentes é uma alternativa de tratamento que permite antecipar a alta hospitalar e o retorno ao ambiente familiar.

(J Pneumol 2001;27(3):148-152)

\section{Home oxygen therapy in children: seven years experience}

Objective: To describe the results of a seven years experience using home oxygen therapy in children with hypoxemia and chronic lung disease. Patients and methods: This is a retrospective and descriptive study carried out at Hospital da Criança Santo Antônio - Porto Alegre-RS, Southern Brazil. The characteristics of the children on home oxygen therapy from January/93 to January/00 were analyzed. Results: Out of the 40 patients studied, 29 (72.5\%) were males. At the beginning of home oxygen therapy, the age of the patients ranged from two months to 13 years and six months (mean: 24.8; median: 13), and 18 of them (45\%) were less than one year old. The most frequent diseases were: obliterative bronchiolitis (31 cases; $77.5 \%)$; chronic obstructive lung disease with aspiration pneumonia (three cases); hypogammaglobulinaemia (two cases); suppurative lung disease (two cases), alveolar proteinosis (one case) and tuberculosis (one case). The length of the last hospitalization before home oxygen therapy was started ranged from nine to 240 days (mean: 63.7 days; median: 50 days). Most of

* Trabalho realizado no Serviço de Pneumologia Pediátrica-Hospital da Criança Santo Antônio, Porto Alegre, RS.

1. Mestre em Pediatria-UFRGS; Médica Pneumologista Pediátrica.

2. Professor Titular da FFFCMPA; Doutor em Pneumologia-UFRGS.

3. Médica Pediatra do Serviço de Pneumologia.
4. Enfermeira da Unidade de Pneumologia.

5. Assistente Social.

Endereço para correspondência - Helena Mocelin, Av. Ceará, 1.549, - 90240-512 - Porto Alegre, RS. Tel./fax (51) 330-6558.

Recebido para publicação em 23/8/00. Reapresentado em 14/ 11/00. Aprovado, após revisão, em 20/2/01. 
the patients $(30 ; 75 \%)$ received oxygen from a concentrator and the remainder from a cylinder. Mean oxygen therapy length ranged from 27 to 1,620 days (median: 392.5 days). There were five deaths from complications of the basic disease and one from accidental removal of the tracheostomy tube. Oxygen use could be discontinued in 19 patients - 15 with obliterative bronchiolitis, two with chronic aspiration pneumonia, one with tuberculosis and one with alveolar proteinosis. Conclusion: Although oxygen was needed for long periods, evolution was satisfactory in most children. Home oxygen for oxygen-dependent is an alternative treatment that allows an early discharge and a quick return to home life.

Descritores - Anoxemia. Pneumopatias obstrutivas. Tratamento domiciliar.

Key words - Oxygen inahalation therapy. Anoxemia. Lung obstructive diseases. Home treatment.

\section{INTRODUÇÃO}

A suplementação de oxigênio por tempo prolongado melhora o prognóstico e a sobrevida de pacientes com hipoxemia crônica ${ }^{(1,2)}$. Atualmente, com os avanços obtidos na terapia intensiva neonatal e pediátrica, a sobrevida de crianças com prematuridade extrema vem aumentando, bem como a daquelas com insuficiência respiratória de causas diversas, como bronquiolite viral aguda, bronquiolite obliterante e fibrose cística, que determinam, muitas vezes, a necessidade de oxigênio suplementar por períodos prolongados. Por outro lado, estudos demonstram que lactentes e crianças com doença pulmonar crônica que, aparentemente, não necessitam de oxigênio em repouso, poderão tornar-se hipoxêmicos durante a alimentação e o sono(3,4). Segundo Hudak et al., a manutenção de oxigenação adequada (saturação de oxigênio da hemoglobina $(\mathrm{SatHb})>$ ou $=95 \%$ ) está associada a maior ganho ponderal e a diminuição da morbidade nesses pacientes ${ }^{(4)}$.

Tem-se observado que pacientes pneumopatas crônicos dependentes de oxigênio suplementar permanecem hospitalizados por tempo prolongado apenas pela necessidade de oxigenoterapia ${ }^{(5)}$. Isso implica alto custo para o sistema de saúde de países em desenvolvimento, custo este que pode ser reduzido através da implementação de adequado programa de fornecimento de oxigênio em nível domiciliar. Além disso, outras complicações graves relacionadas à hospitalização prolongada, como infecção hospitalar e o afastamento do ambiente familiar, poderão ser evitadas ${ }^{(5,6)}$. A literatura tem apresentado resultados satisfatórios com esse tipo de tratamento em adultos com hipoxemia crônica ${ }^{(1,2,7-9)}$, mas pouco tem sido publicado sobre esta alternativa terapêutica em crianças hipoxêmicas.
Este estudo tem por objetivo descrever as características de crianças com hipoxemia crônica, decorrente de doença pulmonar, que integram um programa de oxigenoterapia domiciliar.

\section{MATERIAL E MÉTODOS}

Avaliaram-se, retrospectivamente, 40 crianças em acompanhamento no Serviço de Pneumologia do Hospital da Criança Santo Antônio, Porto Alegre (HCSA), que receberam oxigenoterapia em nivel domiciliar, no período de janeiro de 1993 a janeiro de 2000. Os dados foram obtidos através de revisão de prontuários.

No Serviço de Pneumologia do HCSA os pacientes são incluídos no programa de oxigenoterapia domiciliar se preenchem os seguintes critérios:

Avaliação clínica: 1) história clínica de pneumopatia com hipoxemia crônica (SatHb $<90 \%$ em ar ambiente) ou com dessaturação grave durante o sono (SatHb $<90 \%$ durante um período maior que $10 \%$ do tempo). Para a avaliação da saturação utilizou-se um oxímetro de pulso marca Ohmeda 3700; 2) estabilidade hemodinâmica evidenciada por avaliação médica; e 3) o paciente não pode necessitar de medicações e/ou procedimentos que não possam ser oferecidos em nível domiciliar, tais como medicação intravenosa, fisioterapia intensiva, etc.

Visita domiciliar: realização de visita domiciliar pela enfermeira e assistente social da equipe, que avaliam as condições do domicílio quanto à disponibilidade e características da instalação elétrica e local adequado para a instalação do aparelho, a fim de permitir o uso seguro do equipamento fornecedor de oxigênio. Avalia-se também a capacidade da família de adesão ao tratamento.

Disponibilidade de equipamento para o fornecimento de oxigênio: Aparelho concentrador de oxigênio ou cilindro com válvula, além de um cilindro sobressalente para transporte ou intercorrências.

Os aparelhos são obtidos através de campanhas comunitárias. A sensibilização da família e de eventuais associações ou recursos comunitários faz-se desde o momento em que se percebe que o paciente é candidato à oxigenoterapia domiciliar. 


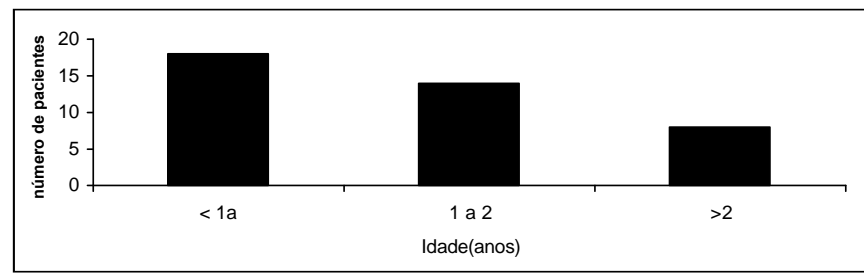

Figura 1 - Idade no início de oxigenoterapia no domicílio

A estratégia de retirada do oxigênio consiste na suspensão deste por pequenos períodos (15, 30 e 60 minutos a cada três horas), quando o paciente apresentar saturação em ar ambiente de $92 \%$. Retira-se o oxigênio noturno somente após a realização de oximetria de pulso durante o sono.

Análise estatística: Os dados foram armazenados e analisados no programa Epi-Info versão 6.0. Realizou-se uma análise descritiva.

\section{REsUlTADOS}

Um total de 40 crianças recebeu oxigenoterapia domiciliar para tratamento de hipoxemia decorrente de doença pulmonar entre janeiro de 1993 e janeiro de 2000. Em 30 (75\%) pacientes o fornecimento de oxigênio foi por aparelho concentrador de oxigênio e, em dez, através de cilindros com válvula. Os aparelhos de oxigênio foram obtidos através de campanhas comunitárias devido às precárias condições socioeconômicas das famílias.

Dos 40 pacientes, $29(72,5 \%)$ eram do sexo masculino. A média de idade em que as crianças iniciaram o uso de oxigênio no domicílio foi de 24,8 meses (mediana: 13 meses) e variou de dois meses a 13 anos e seis meses, sendo que 18 (45\%) eram menores de um ano. A distribuição por faixa etária pode ser observada na Figura 1.

TABELA 1

Freqüência das doenças de base e co-morbidades associadas

\begin{tabular}{|c|c|c|c|}
\hline Bronquiolite obliterante & 31 & $\begin{array}{l}\text { RGE } \\
\text { Traqueostomia } \\
\text { Nanismo } \\
\text { Laringomalacia }\end{array}$ & $\begin{array}{r}12 \\
4\end{array}$ \\
\hline Pneumopatia aspirativa + RGE & 3 & $\begin{array}{l}\text { S. West } \\
\text { Mucopolissacaridose } \\
\text { RDNPM }\end{array}$ & \\
\hline Hipogamaglobulinemia & 2 & $\begin{array}{l}\text { Bronquiectasias } \\
\text { Cor pulmonale }\end{array}$ & 2 \\
\hline Doença pulmonar supurativa crônica & 2 & & \\
\hline Proteinose alveolar & 1 & & \\
\hline Tuberculose & 1 & & \\
\hline
\end{tabular}

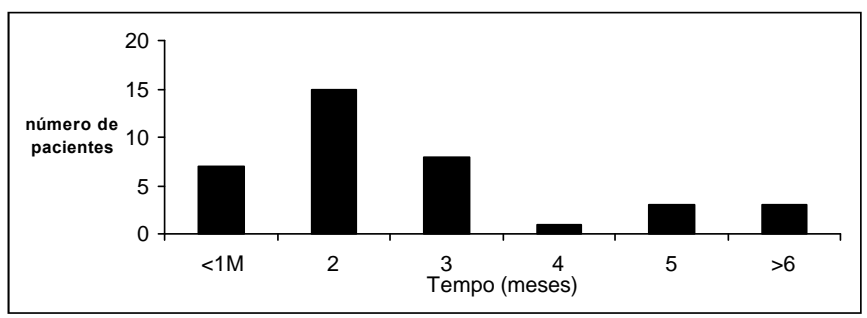

Figura 2 - Tempo de duração da última internação antes da oxigenoterapia

Em relação às doenças de base, em 31 crianças $(77,5 \%)$ foi diagnosticada como bronquiolite obliterante; em três, pneumopatia obstrutiva crônica secundária à aspiração; em duas, hipogamaglobulinemia; em duas, doença pulmonar supurativa crônica; proteinose alveolar e tuberculose, um caso cada. Vários pacientes apresentavam mais de um diagnóstico: refluxo gastroesofágico em 15, cor pulmonale, síndrome de Noonam, mucopolissacaridose, síndrome de Williams, laringomalacia e nanismo em um caso cada. Quatro pacientes eram traqueostomizados ( $\mathrm{Ta}$ bela 1).

O tempo da última internação antes de iniciar oxigênio domiciliar variou de nove a 240 dias (média: 63,7 dias; mediana, 50 dias). A distribuição do tempo de internação pode ser observada na Figura 2. Em três crianças a decisão de oxigenoterapia foi para pacientes em acompanhamento ambulatorial.

As crianças usaram oxigênio no domicílio por um período de 27 dias a 1.620 dias, mediana de 392,5 dias.

A Tabela 2 apresenta a evolução dos pacientes. Em 19 $(48,7 \%)$ crianças o oxigênio foi suspenso por melhora clínica e, nestes, o diagnóstico foi de bronquiolite obliterante em 15 casos, pneumonia aspirativa em dois, tuberculose em um e proteinose alveolar em outro. Ocorreram seis óbitos: hipogamaglobulinemia em dois, doença pulmonar supurativa crônica e pneumonectomia em um, síndrome de West com pneumonia aspirativa em outro; em uma criança o óbito foi associado à retirada acidental da cânula de traqueostomia e, em outra, que já não necessitava mais de suplementação de oxigênio, o óbito foi devido a sepse.

TABELA 2

Evolução dos pacientes com oxigenoterapia domiciliar

\begin{tabular}{lc}
\hline \multicolumn{1}{c}{ Evolução } & Número de pacientes \\
\hline Suspensão do $\mathrm{O}_{2}$ & 19 \\
Usando $\mathrm{O}_{2}$ & 15 \\
Óbito & 6 \\
\hline
\end{tabular}




\section{DISCUSSÃO}

Os benefícios da oxigenoterapia domiciliar são conhecidos há mais de 40 anos. Atualmente, com o advento dos concentradores de oxigênio, esta tornou-se a forma mais simples e econômica de fornecimento de oxigênio a longo prazo(2,10).

Dados na literatura demonstram que adultos com hipoxemia crônica, que recebem suplementação de oxigênio, têm redução nas hospitalizações, apresentam melhora na sobrevida, na capacidade de exercício e no desenvolvimento neuropsicológico(11,12). Estudos indicam que a reversão da hipoxemia em pacientes com doença pulmonar obstrutiva crônica (DPOC) é responsável pela redução da mortalidade quando o oxigênio é administrado por no mínimo 15 horas/dia ${ }^{(10)}$. Em crianças com displasia broncopulmonar a oxigenoterapia promove crescimento somático e neurológico adequado e previne o desenvolvimento de hipertensão pulmonar ${ }^{(4,10)}$.

Embora várias pesquisas realizadas em adultos com DPOC demonstrem os benefícios da terapia a longo prazo com oxigênio ${ }^{(9,10)}$, encontramos poucos estudos em crianças com hipoxemia crônica que receberam oxigênio após a alta hospitalar. Neste estudo, que avaliou as características de pacientes na faixa etária pediátrica com oxigenoterapia em nível domiciliar, pode-se observar que as crianças apresentaram boa evolução, apesar de a maioria necessitar de oxigênio por períodos prolongados.

Segundo alguns autores, a indicação de oxigênio suplementar deve ser baseada em dados de gasometria arterial(10,13). Outros baseiam-se na medida da SatHb que, embora menos precisa, se correlaciona com a $\mathrm{PaO}_{2}^{(2,14)}$. Neste serviço, a oximetria de pulso é utilizada como principal parâmetro para a indicação de oxigenoterapia, por ser um método não invasivo, de baixo custo e disponível para monitorização ambulatorial.

A oxigenoterapia a longo prazo pode ser administrada por aparelho concentrador de oxigênio ou sob a forma de oxigênio líquido ou gás comprimido. A vantagem dos concentradores, quando comparados com as demais formas de administração de oxigênio, é que eles são relativamente baratos, requerem pouca manutenção e não necessitam de reposição de carga, ao contrário dos cilindros. Neste estudo, os equipamentos de fornecimento de oxigênio foram utilizados conforme a disponibilidade dos aparelhos. Considerando-se que nesta série de pacientes as condições socioeconômicas eram precárias, foi necessário utilizar de campanhas comunitárias para a aquisição dos mesmos. Embora com dificuldades financeiras, a maioria das famílias teve condições de manter as crianças no domicílio.

Das 40 crianças arroladas no estudo, a maioria era do sexo masculino $(72,5 \%)$, semelhante ao encontrado por
Fauroux et al. (62\%)(15). Estes achados estão de acordo com os dados da literatura, que demonstram que os meninos são acometidos com maior freqüência e gravidade por doenças respiratórias ${ }^{(16)}$. Observamos também que a maioria das crianças iniciou o uso de oxigênio muito precocemente, $56 \%$ antes do primeiro ano de vida. Este achado pode estar relacionado com a freqüência de bronquiolite obliterante, que incidiu em $77,5 \%$ dos pacientes estudados, sendo a principal enfermidade responsável pela hipoxemia crônica nesta amostra. Bronquiolite obliterante é a seqüela mais grave de bronquiolite viral aguda; predomina nos meses de inverno nos locais onde as estações do ano são bem definidas, como no Rio Grande do Sul, onde crianças menores de seis meses são acometidas com maior freqüência. As crianças com bronquiolite obliterante tendem a apresentar altas taxas de reinternação, uso prolongado de $\mathrm{O}_{2}$ e alta prevalência de bronquiectasias $^{(17)}$. A hipoxemia é um achado freqüente nesses pacientes, embora, na maioria das vezes, seja possível manter saturação adequada com a suplementação de oxigênio em baixas concentrações, o que se consegue com qualquer um dos métodos disponíveis para fornecimento de oxigênio. No HCSA, encontram-se em acompanhamento 85 crianças com diagnóstico de bronquiolite obliterante, sendo que 31 usaram oxigênio no domicílio(5,6). Bertrand et al. observaram que crianças com doença respiratória neonatal e displasia broncopulmonar tiveram melhor prognóstico e o oxigênio foi descontinuado após quatro e seis meses, respectivamente $e^{(18)}$. Hudak et al. também observaram média de 4,5 meses de uso de oxigênio em crianças com displasia broncopulmonar ${ }^{(4)}$.

As internações prolongadas (média de 63 dias) refletem a gravidade e a alta morbidade nos pacientes estudados. Ressalte-se que em muitas situações as crianças permanecem hospitalizadas até a obtenção do equipamento que lhes possibilite a alta. Nestes casos, deve-se considerar o alto custo para o sistema de saúde gerado por essas internações que tem sido observado em outros estudos ${ }^{(18)}$.

Considerando-se o tempo de uso de oxigênio domiciliar, evitaram-se 16.984 dias de hospitalização; estima-se que o sistema de saúde gastaria cerca de $\mathrm{R} \$ 186.824,00$ com a permanência dessas crianças no hospital.

Embora, por questões éticas, não se tenha um grupo controle para os pacientes estudados, pode-se observar uma evolução satisfatória dessas crianças oxigenodependentes. Mesmo que a maioria dos pacientes tivesse como diagnóstico bronquiolite obliterante, houve apenas um óbito devido à retirada acidental da cânula de traqueostomia. Os dados obtidos sugerem que o prognóstico de crianças oxigenodependentes esteja associado com a doença de base.

Desde 1993, o acompanhamento dos pacientes oxigenodependentes do nosso serviço é realizado por uma equi- 
pe multidisciplinar que inclui médicos, enfermeira, psicóloga, nutricionista e assistente social, que juntos trabalham para aquisição de equipamentos, treinamento $e$ orientação dos pacientes. Acreditamos que o sucesso da oxigenoterapia domiciliar somente será possivel se os serviços de saúde que atendem este tipo de paciente criarem um programa específico de assistência(19).

Através deste estudo concluiu-se que, apesar da necessidade de oxigênio por períodos prolongados, a evolução da maioria dos pacientes oxigenodependentes é satisfatória. Oxigênio domiciliar é uma alternativa de tratamento que permite antecipar a alta hospitalar, propiciando o retorno da criança ao seu ambiente familiar, além de redução substancial dos custos de internação para o sistema de saúde.

\section{REFERÊNCIAS}

1. Dilworth JP, Higgs CMB, Jones PA, White. Prescription of oxygen concentrators: adherence to published guidelines. Thorax 1989;44: 576-478.

2. Tarpy SP, Celli BR. Long-term oxygen therapy. N Engl J Med 1995; 14:710-714.

3. Poets CF. When do infants need additional inspired oxygen? A review of the current literature. Pediatr Pulmonol 1998;26:424-428.

4. Hudak BB, Allen MC, Hudak ML, Loughlin GM. Home oxygen therapy for chronic lung disease in extremely low-birth-weight infants. Am J Dis Child 1989;143:357-360.

5. Mocelin HT, Rosa DR, Oliveira SK, Fischer GB. Oxigenoterapia domiciliar em pneumopatias crônicas. Anais IV Congreso de la Sociedad Latinoamericana de Neumologia Pediatrica, 1998; trabajo libre 116.

6. Mocelin HT, Souza MV, Ranzi LC, Oliveira R, Philomena MR, Fischer GB. Oxigenoterapia domiciliar - Relato de 5 anos de experiência. Anais do VIII Congresso Brasileiro de Pneumologia Pediátrica, 1999; poster 092; p. 168.

7. Restrick LJ, Paul EA, Braid GM, Cullinan, Moore-Gillon J, Wedzicha JA. Assessment and follow-up of patients prescribed long-term oxygen treatment. Thorax 1993;48:708-713.

8. McKeon J, Murree-Allen K, Saunders NA. Supplemental oxygen and quality of sleep in patients with chronic obstructive lung disease. Tho$\operatorname{rax} 1989 ; 44: 184-188$

9. Ström K. Survival of patients with chronic obstructive pulmonary disease receiving long-term domiciliary oxygen therapy. Am Rev Respir Dis 1993; 147:585-591.

10. Petty TL. Oxigênio domiciliar: uma revolução no tratamento da DPOC avançada. Clín Méd Am Norte 1990;3:751-766.

11. Nocturnal Oxygen Therapy Trial Group. Continuous or nocturnal oxygen therapy in hypoxemic chronic obstrutive lung disease. Ann Intern Med 1980;93:391-398.

12. Skwarski K, MacNee W, Wraith KP, Sliwinski P, Zielinski J. Predictors of survival in patients with chronic obstrutive pulmonary disease treated with long-term oxygen therapy. Chest 1991;100:1522-1527.

13. Barker AF, Burgher LW, Plummer AL. Oxygen conserving methods for adults. Chest 1994;105:248-252.

14. Decker MJ, Arnold LJ, Haney D, Masny J, Strohl PK. Extended monitoring of oxygen saturation in chronic lung disease. Chest 1992;102: 1075-1079.

15. Fauroux B, Sardet A, Foret D. Home treatment for chronic respiratory failure in children: a prospective study. Eur Respir J 1995;8:12,20622066.

16. Fischer GB. Fatores prognósticos para bronquiolite viral aguda. [Tese] Porto Alegre: Universidade Federal do Rio Grande do Sul, 1994.

17. Fischer GB, Mocelin HT. Bronquiolite obliterante - Seqüelas de bronquiolite. In: Rozov T. Doenças pulmonares em pediatria: diagnóstico e tratamento. São Paulo: Atheneu 1999;199-204.

18. Bertrand P, Alvarez C, Fabres J, Simonetti M, Sánchez I. Home oxygen therapy in children with chronic respiratory failure. Rev Med Chil 1998; 126:284-292.

19. Petty TL, Casaburi R. Recommendations of the Fifth Oxygen Consensus Conference. Respir Care 2000;45:957-961. 